\title{
Estudo do seǵmento anterior com a câmera rotatória de Scheimpflug em pacientes candidatos à cirurgia refrativa
}

\author{
Anterior ocular segment study with the Scheimpflug rotational camera \\ in refractive surgerycandidates
}

\author{
Celina Murata ${ }^{1}$ \\ Felipe Mallmann² \\ Ester Yamazaki ${ }^{3}$ \\ Mauro Campos ${ }^{4}$
}

Trabalho realizado no Setor de Cirurgia Refrativa do Departamento de Oftalmologia da Universidade Federal de São Paulo - UNIFESP - São Paulo (SP) - Brasil.

${ }^{1}$ Médica Oftalmologista do Setor de Cirurgia Refrativa do Departamento de Oftalmologia da Universidade Federal de São Paulo - UNIFESP - São Paulo (SP) - Brasil. Médico Oftalmologista do Setor de Cirurgia Refrativa do Departamento de Oftalmologia da UNIFESP - São Paulo (SP) - Brasil.

${ }^{3}$ Médica Oftalmologista do Setor de Cirurgia Refrativa do Departamento de Oftalmologia da UNIFESP - São Paulo (SP) - Brasil.

${ }^{4}$ Professor Adjunto do Departamento de Oftalmologia da UNIFESP - São Paulo (SP) - Brasil.

Endereço para correspondência: Celina Murata. Rua Borges Lagoa, 368 - São Paulo (SP) CEP 04038-000 E-mail: celmurata@yahoo.com.br

Recebido para publicação em 25.10.2006

Última versão recebida em 20.04.2007

Aprovação em 30.04.2007

Nota Editorial: Depois de concluída a análise do artigo sob sigilo editorial e com a anuência da Dra. Adriana dos Santos Forseto sobre a divulgação de seu nome como revisora, agradecemos sua participação neste processo.

\section{RESUMO}

Objetivo: Analisar o segmento anterior com aparelho de câmera rotatória de Scheimpflug $\left(\right.$ Pentacam $\left.^{\circledR}\right)$ em candidatos à cirurgia refrativa, estabelecendo o padrão de variabilidade do volume corneano, do volume e da profundidade da câmara anterior e da paquimetria óptica nesta população. Métodos: Estudo retrospectivo de 297 olhos (149 pacientes) que foram examinados com Pentacam ${ }^{\circledR}$. Os pacientes foram divididos conforme o equivalente esférico em míopes ( $n=242$ olhos) e hipermétropes $(n=55$ olhos) para analisar o volume corneano, o volume e a profundidade da câmara anterior e a paquimetria óptica. Resultados: No grupo dos míopes, a média do volume corneano total foi de $59,37 \pm 3,51 \mathrm{~mm}^{3}$; do volume nos $3 \mathrm{~mm}$ de $3,87 \pm 0,23 \mathrm{~mm}^{3}$, nos $5 \mathrm{~mm}$ de $11,31 \pm 0,67 \mathrm{~mm}^{3}$, e nos $7 \mathrm{~mm}$ de 24,30 $\pm 1,43 \mathrm{~mm}^{3}$; do volume da câmara anterior $198,74 \pm 32,40 \mathrm{~mm}^{3}$; da profun-

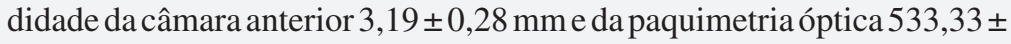
$33,47 \mu \mathrm{m}$. No grupo dos hipermétropes, a média do volume corneano total foi de $60,77 \pm 3,31 \mathrm{~mm}^{3}$, do volume nos $3 \mathrm{~mm} \mathrm{de} 4,01 \pm 0,20 \mathrm{~mm}^{3}$, nos $5 \mathrm{~mm}$ de $11,73 \pm 0,58 \mathrm{~mm}^{3}$, e nos $7 \mathrm{~mm}$ de $25,09 \pm 1,21 \mathrm{~mm}^{3}$; do volume da câmara anterior $146,61 \pm 32,86 \mathrm{~mm}^{3}$; da profundidade da câmara anterior 2,76 \pm $0,38 \mathrm{~mm}$ e da paquimetria de $550,52 \pm 29,49 \mu \mathrm{m}$. A diferença entre os grupos foi significativa para todas as variáveis $(\mathrm{p}<0,05)$. Conclusão: Concluímos nesta amostra, por meio da análise pelo Pentacam ${ }^{\circledR}$, que os olhos míopes apresentaram menor média de volume corneano e paquimetria óptica, e maior profundidade e volume de câmara anterior em relação aos hipermétropes.

Descritores: Topografia da córnea; Miopia/cirurgia; Hiperopia/cirurgia; Segmento anterior do olho

\section{INTRODUÇÃO}

A transparência corneana é essencial para a visão e um endotélio funcionalmente intacto é necessário para a manutenção do estroma claro através da regulação da hidratação da córnea ${ }^{(1)}$.

A medida da espessura corneana é um indicador importante da função endotelial $^{(2-3)}$. A paquimetria é utilizada na programação de cirurgias refrativas, na evolução da descompensação endotelial e no pós-operatório de cirurgias intra-oculares ${ }^{(2)}$.

O aparelho Pentacam ${ }^{\circledR}$ (Oculus, Wetzlar, Alemanha) usa uma câmera rotacional de Scheimpflug para a visualização do segmento anterior do olho. É um instrumento de não contato, que fornece em um único escaneamento várias imagens dos $360^{\circ}$ do segmento anterior em duas e três dimensões, gerando mapas de topografia anterior e posterior da córnea, de paquimetria, além de medidas da câmara anterior e análise do cristalino, 
como densitometria de opacidades cristalinianas ${ }^{(4)}$. A espessura corneana é determinada por medidas tomográficas da superfície anterior e posterior da córnea realizadas pela câmera rotacional ${ }^{(5)}$.

Alguns trabalhos compararam as medidas de paquimetria realizadas pela câmera rotacional de Scheimpflug, pelo topógrafo de escaneamento em fenda (Orbscan ${ }^{\circledR}$, Orbtek Inc, Salt Lake City, Utah, Estados Unidos), pela interferometria de coerência parcial (AC-Master ${ }^{\circledR}$, Zeiss Meditec, Jena Alemanha), pela reflectometria de baixa coerência (Haag-Streit) ou pelo paquímetro ultra-sônico ${ }^{(4-6)}$. Contudo, a literatura carece de dados a respeito das medidas de volume da córnea e volume e profundidade de câmara anterior.

Nosso objetivo é analisar o segmento anterior com o aparelho de câmera rotatória de Scheimpflug, em pacientes candidatos à cirurgia refrativa, quanto ao volume da córnea $(3 \mathrm{~mm}, 5 \mathrm{~mm}$, $7 \mathrm{~mm}$ e $10 \mathrm{~mm}$ ), volume e profundidade da câmara anterior e espessura corneana, procurando estabelecer o padrão de variabilidade destas medidas na população em estudo.

\section{MÉTODOS}

Foi realizado um estudo retrospectivo do prontuário dos pacientes candidatos à cirurgia refrativa atendidos no ambulatório de Cirurgia Refrativa do Departamento de Oftalmologia da Escola Paulista de Medicina. Foram revisados dados de refração sob cicloplegia, tonometria, biomicroscopia e exame de Pentacam ${ }^{\circledast}$. Foram excluídos pacientes com ambliopia, ceratocone, glaucoma, cirurgia ocular prévia ou qualquer opacidade corneana que pudesse interferir na fixação do exame. Os pacientes recebem, de rotina, a orientação para não usarem lentes de contatos por pelo menos 1 semana antes da primeira consulta.

No exame do Pentacam ${ }^{\circledR}$, durante a aquisição da imagem, o paciente é instruído a fixar em um alvo no centro da câmera. $\mathrm{O}$ examinador tem a imagem em tempo real do olho do paciente na tela do computador e a imagem deve ser centrada e focada manualmente. Assim que a imagem é perfeitamente alinhada, a câmera rotatória tira múltiplas imagens em fenda do segmento anterior do olho ( 25 ou 50, dependendo da escolha do examinador) em aproximadamente 2 segundos com 500 pontos de elevação verdadeira incorporados em cada imagem em fenda. Após completar o escaneamento, o programa calcula uma imagem tridimensional do segmento anterior ${ }^{(7)}$.

A medida do volume total da córnea pelo Pentacam $^{\circledR}$ é calculada a partir do ápice num diâmetro de $10 \mathrm{~mm}$. O aparelho calcula também o volume nos diâmetros de $3 \mathrm{~mm}, 5 \mathrm{~mm}$ e $7 \mathrm{~mm}$ a partir do ponto mais fino ${ }^{(7)}$.

O volume da câmara anterior é calculado a partir da distância da superfície posterior da córnea, da íris e do cristalino integrados num diâmetro de $12 \mathrm{~mm}$ em torno do ápice corneano. A profundidade da câmara anterior a partir do ápice corneano até a superfície anterior do cristalino é calculada num modelo tridimensional, sendo o ponto de referência considerado neste estudo o endotélio, sendo possível o cálculo também pelo epitélio ${ }^{(6-7)}$.
Quanto aos dados de paquimetria óptica, foram estudadas as medidas da córnea no local mais fino.

Estes pacientes foram divididos em dois grupos conforme o equivalente esférico (míopes e hipermétropes) para analisar as variáveis de volume corneano, volume e profundidade da câmara anterior e paquimetria óptica e avaliar a diferença entre os grupos.

O grupo dos míopes foi ainda subdividido em 4 grupos conforme a ametropia: grupo 1 ( 0 a -3, 0 D), grupo $2(-3,1$ a $-6 D)$, grupo 3 (-6,1 a -9 D) e grupo 4 (>-9 D), para avaliar a diferença entre eles quanto às variáveis analisadas.

Foi realizada a comparação dos grupos dos míopes e dos hipermétropes como um todo, depois foram comparadas ametropias semelhantes entre estes dois grupos (míopes de 0 a $-6,0$ D e hipermétropes de $+0,1$ a +6,0 D).

Os exames foram realizados por examinadores treinados, observando os parâmetros de qualidade determinados pelo fabricante.

A análise de médias, desvio padrão e a construção de gráficos foram realizadas no programa estatístico Statistical Package for Social Sciences versão 11.0 (SPSS Inc, Chicago, Ilinois). Resultados com erro alfa (p) menor que 0,05 foram considerados significativos. As variáveis foram analisadas conforme sua distribuição seguindo a curva normal, sendo realizado também o teste de Komolgorov-Smirnov. O teste $\mathrm{t}$ independente foi utilizado para comparação daquelas variáveis que seguiam a curva normal e o teste de Mann-Whitney para as com distribuição diferente desta. Foi utilizado também o teste ANOVA para a comparação das médias entre os grupos.

\section{RESULTADOS}

Foram estudados no total 149 pacientes (297 olhos). Destes, 242 olhos eram míopes e 55 olhos hipermétropes. Um olho de uma paciente foi excluído devido à dificuldade na fixação e realização do exame (ambliopia). Três pacientes tinham um olho míope e o outro hipermétrope.

No grupo dos míopes, $78(63,4 \%)$ pacientes eram do sexo feminino, a idade média foi de 32,61 $\pm 8,91$ anos (variação de 21 a 59 anos). Quanto à cor, $110(89,4 \%)$ eram brancos, 4 (3,3\%) pretos, $5(4,1 \%)$ amarelos e 4 (3,3\%) pardos. Dos olhos míopes analisados, $120(49,6 \%)$ eram olhos direitos. A média do equivalente esférico foi de $-3,53 \pm 2,48 \mathrm{D}$ (variação de 0 a - 15,25 D).

Quanto às variáveis analisadas no grupo dos míopes, as médias dos volumes corneanos foram: total de $59,37 \pm 3,51 \mathrm{~mm}^{3}$ (variação de 50,6 a 68,10 $\mathrm{mm}^{3}$ ), nos $3 \mathrm{~mm}$ de $3,87 \pm 0,23 \mathrm{~mm}^{3}$ (variação de 3,20 a $4,50 \mathrm{~mm}^{3}$ ), nos $5 \mathrm{~mm}$ de $11,31 \pm 0,67 \mathrm{~mm}^{3}$ (variação de 9,40 a $13 \mathrm{~mm}^{3}$ ), e nos $7 \mathrm{~mm}$ de $24,30 \pm 1,43 \mathrm{~mm}^{3}$ (variação de 20,30 a 27,50 $\mathrm{mm}^{3}$ ). Quanto ao volume da câmara anterior, a média foi de $198,74 \pm 32,40 \mathrm{~mm}^{3}$ (variação de 117 a $286 \mathrm{~mm}^{3}$ ) e em relação à profundidade da câmara anterior a média foi de $3,19 \pm 0,28 \mathrm{~mm}$ (variação de 2,48 a 3,89 mm). A média da paquimetria óptica foi de 533,33 $\pm 33,47 \mu \mathrm{m}$ (variação de 444 a $617 \mu \mathrm{m})$ (Tabela 1). 
$\mathrm{Na}$ subdivisão por quantidade de ametropia dentro do grupo dos míopes, diferenças estatisticamente significativas foram observadas quanto ao volume de $3 \mathrm{~mm}(\mathrm{p}=0,015)$, volume de $5 \mathrm{~mm}(\mathrm{p}=0,021)$, volume de câmara anterior $(\mathrm{p}=0,037)$, profundidade de câmara anterior $(\mathrm{p}=0,025)$ e paquimetria óptica $(\mathrm{p}=0,015)$ (Tabela 2$)$.

No grupo dos hipermétropes, 18 (62\%) eram do sexo feminino, a idade média foi de 40,30 $\pm 11,40$ anos (variação de 20 a 55 anos). Quanto à cor, todos eram brancos. Dos 55 olhos analisados, $29(52,7 \%)$ eram olhos direitos. O equivalente esférico médio foi de $+3,56 \pm 2,15 \mathrm{D}$ (variação de $+0,25 \mathrm{a}+9,25 \mathrm{D}$ ).

A comparação da média de idade entre os míopes e hipermétropes mostrou uma diferença significativa entre os grupos (32,61 anos versus 40,30 anos; $\mathrm{p}<0,001)$, mostrando que os míopes eram mais jovens que os hipermétropes neste estudo. Não houve diferença estatisticamente significativa quanto à idade entre os subgrupos dos míopes ( $\mathrm{p}=0,097)$.

Quanto às variáveis analisadas no grupo dos hipermétropes, as médias dos volumes corneanos foram: total de 60,77 \pm $3,31 \mathrm{~mm}^{3}$ (variação de 52,50 a $66,80 \mathrm{~mm}^{3}$ ), nos $3 \mathrm{~mm}$ de $4,01 \pm$ $0,20 \mathrm{~mm}^{3}$ (variação de 3,50 a 4,40 $\mathrm{mm}^{3}$ ), nos $5 \mathrm{~mm}$ de 11,73 \pm $0,58 \mathrm{~mm}^{3}$ (variação de 10,10 a 12,60 $\mathrm{mm}^{3}$ ), e nos $7 \mathrm{~mm}$ de 25,09 \pm $1,21 \mathrm{~mm}^{3}$ (variação de 21,80 a $26,70 \mathrm{~mm}^{3}$ ). Quanto ao volume da câmara anterior, a média foi de 146,61 $\pm 32,86 \mathrm{~mm}^{3}$ (variação de $89 \mathrm{a} 249 \mathrm{~mm}^{3}$ ) e em relação à profundidade da câmara anterior a média foi de 2,76 $\pm 0,38 \mathrm{~mm}$ (variação de 2,06 a 3,98 mm). A média da paquimetria óptica foi de $550,52 \pm 29,49 \mu \mathrm{m}$ (variação de 475 a $606 \mu \mathrm{m}$ ) (Tabela 1 ).
Como podemos observar na tabela 1 , todas as médias de volume corneano, volume e profundidade de câmara anterior e paquimetria óptica mostraram uma diferença estatisticamente significativa entre o grupo dos míopes e hipermétropes $(\mathrm{p}<0,05)$.

A análise comparativa dos míopes e dos hipermétropes de ametropias semelhantes mostrou uma diferença significativa para o volume de $3 \mathrm{~mm}$, de $5 \mathrm{~mm}$, de $7 \mathrm{~mm}$, o volume e a profundidade de câmara anterior e a paquimetria óptica (Tabela 3).

Quanto ao uso de lente de contato (LC), observou-se que somente os míopes eram usuários. Foi possível colher dados a respeito do uso de LC somente de 67 olhos $(24,4 \%$ do total de olhos do estudo). Destes, 63 olhos (94\%) usavam LC gelatinosas. A média do uso de $\mathrm{LC}$ destes pacientes foi de 76,68 \pm 47,45 meses (variação de 1 a 192 meses). Quanto ao tempo de uso de LC nos subgrupos dos míopes, no grupo 1 a média de uso foi de $65,58 \pm$ 44,14 meses (29 pacientes); no grupo 2, 82,84 $\pm 49,95$ meses (38 pacientes); no grupo 3, $57 \pm 45,29$ meses (4 pacientes); não havia dados de pacientes do grupo 4 quanto ao uso de LC. Não houve diferença estatisticamente significativa entre os grupos.

\section{DISCUSSÃO}

Neste estudo, os míopes, em comparação com os hipermétropes, apresentaram menor volume corneano total $(\mathrm{p}=0,008)$, $\operatorname{nos} 3 \mathrm{~mm}(\mathrm{p}<0,001), 5 \mathrm{~mm}(\mathrm{p}<0,001)$ e $7 \mathrm{~mm}(\mathrm{p}<0,001)$. Na análise da subdivisão dos grupos míopes, observou-se que foi significativa a diferença entre os grupos quanto ao volume de $3 \mathrm{~mm}$ $(\mathrm{p}=0,015)$ e de $5 \mathrm{~mm}(\mathrm{p}=0,021)$, sendo que quanto maior a ame-

\begin{tabular}{|c|c|c|c|}
\hline Variáveis & Míopes $(n=242)$ & Hipermétropes $(n=55)$ & $p^{*}$ \\
\hline Volume total $\left(\mathrm{mm}^{3}\right)$ & $59,37 \pm 3,51$ & $60,77 \pm 3,31$ & 0,008 \\
\hline Volume $3 \mathrm{~mm}\left(\mathrm{~mm}^{3}\right)$ & $3,87 \pm 0,23$ & $4,01 \pm 0,20$ & $<0,001$ \\
\hline Volume $5 \mathrm{~mm}\left(\mathrm{~mm}^{3}\right)$ & $11,31 \pm 0,67$ & $11,73 \pm 0,58$ & $<0,001$ \\
\hline Volume $7 \mathrm{~mm}\left(\mathrm{~mm}^{3}\right)$ & $24,30 \pm 1,43$ & $25,09 \pm 1,21$ & $<0,001$ \\
\hline Volume de câmara anterior $\left(\mathrm{mm}^{3}\right)$ & $198,74 \pm 32,40$ & $146,61 \pm 32,86$ & $<0,001$ \\
\hline
\end{tabular}

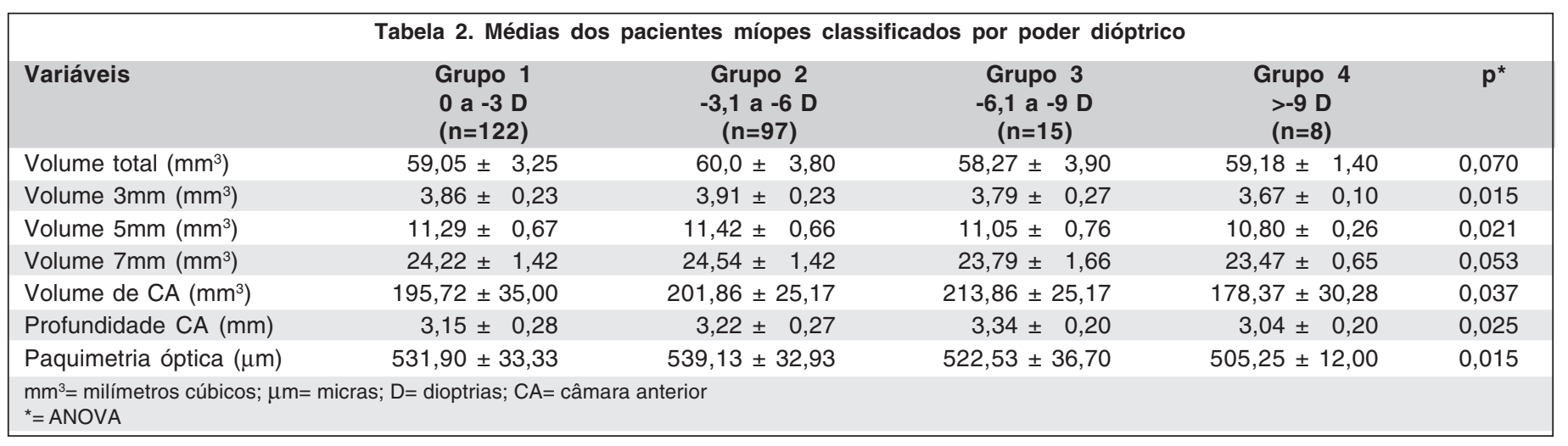


tropia, menor o volume (Tabela 2). Na comparação dos míopes e hipermétropes com ametropias semelhantes (Tabela 3), notouse que os míopes apresentaram volume corneano menor nos $3 \mathrm{~mm}(\mathrm{p}=0,002), 5 \mathrm{~mm}(\mathrm{p}=0,002)$ e $7 \mathrm{~mm}(\mathrm{p}=0,002)$. Observa-se, com isso, um menor volume corneano ao redor do ápice principalmente nos $3 \mathrm{~mm}, 5 \mathrm{~mm}$ e $7 \mathrm{~mm}$ em pessoas míopes.

Existem alguns estudos na literatura sobre volume corneano em cirurgia de catarata e não há relatos sobre volume em míopes e hipermétropes com o aparelho Pentacam ${ }^{\circledR}$. Um estudo mostrou que o volume corneano medido pelo Pentacam ${ }^{\circledR}$ pode ser útil para avaliar o dano causado ao endotélio devido à cirurgia de facoemulsificação e aspiração em comparação com a microscopia especular $^{(8)}$. Observou-se que o volume corneano aos $10 \mathrm{~mm}$ foi significativamente maior com um mês de pósoperatório em comparação ao pré-operatório, não havendo diferença significativa na medida do volume aos $3 \mathrm{~mm}$ entre o pré e o pós-operatório de um mês. A microscopia especular, geralmente avaliada na área central, representa apenas uma pequena porção do dano endotelial decorrente da cirurgia e o volume corneano estuda uma extensão maior da córnea ${ }^{(8)}$.

Outro estudo avaliou o volume corneano pós-cirurgia de catarata através do aparelho Orbscan ${ }^{\circledR(9)}$. Neste estudo foi avaliado o volume corneano dos $3 \mathrm{~mm}$ centrais no pré-operatório, 6 h e 24 h após a facoemulsificação. O volume aumentou até 6 h, havendo uma diminuição após $24 \mathrm{~h}$, com medidas médias no pré de 4,64 $\mathrm{mm}^{3}$, após $6 \mathrm{~h}$ de 5,36 $\mathrm{mm}^{3}$, e após 24 h de 5,05 $\mathrm{mm}^{3}$. Os autores defendem que o Orbscan ${ }^{\circledR}$ é um bom método nãoinvasivo para se obter o volume corneano; além de considerarem a medida do volume central mais vantajosa que a paquimetria, que pode apresentar diferentes espessuras dependendo da localização, para avaliação da função endotelial ${ }^{(9)}$.

Neste estudo, a média da paquimetria a partir do ponto mais fino foi de 533,33 $\pm 33,47 \mu \mathrm{m}$ para os míopes e de 550,52 \pm 29,49 $\mu \mathrm{m}$ para os hipermétropes, com uma diferença estatisticamente significativa entre os grupos $(\mathrm{p}=0,001)$. A comparação de ametropias semelhantes de miopia e hipermetropia mostrou valores aproximados $(534,88 \pm 33,20 \mu \mathrm{m}$ versus $547,34 \pm$ $28,95 \mu \mathrm{m})$ com uma diferença significativa $(\mathrm{p}=0,018)$. Na subdivisão dos míopes, observou-se que quanto maior a ametropia menos espessa era a paquimetria óptica $(\mathrm{p}=0,015)$. A análise destes dados mostra que os olhos míopes apresentam uma tendência a uma espessura corneana menor em torno do ápice.

As mudanças decorrentes da miopia no globo ocular incluem aumento do comprimento axial, aumento da profundidade da câmara anterior e da câmara vítrea, afinamento retiniano com degenerações periféricas e maior prevalência de descolamento de retina, diminuição da circulação coroidea, diminuição da espessura e da elasticidade escleral. Se o volume corneano não aumenta, espera-se que o estroma corneano se torne mais fino, semelhante ao que acontece com a esclera na progressão da miopia $^{(10)}$. Isso explica o achado de córneas mais finas em olhos míopes. Além disso, vários estudos sustentam esta teoria.

Um estudo realizado em 1956 reportou córneas mais finas em míopes com mais de $-4,0 \mathrm{D}^{(11)}$. Achado semelhante foi descrito em 1976, o autor encontrou uma diferença de $0,018 \mathrm{~mm}$ na espessura de míopes em comparação com os controles normais ${ }^{(12)}$.

A comparação da espessura corneana entre portadores de alta miopia (-9,0 D a -25,0 D) e de baixa ametropia $(-3,7 \mathrm{a}+3,2 \mathrm{D})$ encontrou uma média de paquimetria ultra-sônica de $563,5 \mu \mathrm{m}$ e $581,1 \mu \mathrm{m}$ respectivamente $(\mathrm{p}=0,004)^{(13)}$. Os indivíduos altos míopes parecem apresentar córneas menos espessas do que os emétropes ou portadores de baixas ametropias ${ }^{(13)}$.

Em um estudo realizado na Tailândia, 216 indivíduos com equivalente esférico médio de $-4,17 \pm 5,03 \mathrm{D}$ apresentaram uma medida de paquimetria ultra-sônica média de $533 \pm 29 \mu \mathrm{m}^{(10)}$. As córneas tenderam a ser mais finas em ametropias mais altas $(\mathrm{r}=0,16, \mathrm{p}=0,021)^{(10)}$.

Entretanto, um estudo de medida da espessura central corneana usando o Orbscan ${ }^{\circledR}$ em 714 pacientes chineses com equivalente esférico médio de $-5,30 \pm 2,74 \mathrm{D}$, encontrou uma média de espessura central de 534,5 $\pm 38,1 \mu \mathrm{m}^{(14)}$. Neste estudo não foi encontrada uma correlação entre a espessura central corneana e o grau da miopia $(\mathrm{r}=0,13, \mathrm{p}=0,719)^{(14)}$. Outros estu$\operatorname{dos}^{(15-17)}$ também não mostraram correlação entre a espessura central corneana e o grau de miopia. No entanto, em dois trabalhos ${ }^{(15-16)}$, os pacientes eram usuários de lentes de contato (LC), podendo haver variações individuais após o uso das mesmas, explicando a falta de correlação entre a ametropia e a espessura corneana.

Um estudo mostrou os efeitos corneanos do uso crônico de LC gelatinosas em olhos míopes e hipermétropes ${ }^{(18)}$. O

\begin{tabular}{|c|c|c|c|}
\hline Volume total $\left(\mathrm{mm}^{3}\right)$ & $59,48 \pm 3,53$ & $60,43 \pm 3,30$ & 0,092 \\
\hline Volume $3 \mathrm{~mm}\left(\mathrm{~mm}^{3}\right)$ & $3,88 \pm 0,23$ & $3,99 \pm 0,20$ & 0,002 \\
\hline Volume $5 \mathrm{~mm}\left(\mathrm{~mm}^{3}\right)$ & $11,34 \pm 0,67$ & $11,68 \pm 0,58$ & 0,002 \\
\hline Profundidade CA (mm) & $3,18 \pm 0,28$ & $2,71 \pm 0,33$ & $<0,001$ \\
\hline Paquimetria óptica $(\mu \mathrm{m})$ & $534,88 \pm 33,20$ & $547,34 \pm 28,95$ & 0,018 \\
\hline
\end{tabular}


tempo médio de uso das LC foi de $62 \pm 29$ meses e observou-se uma redução significativa da espessura epitelial e estromal de $5,6 \%$ e $2,3 \%$ respectivamente, comparado aos controles. Além disso, foram constatados uma redução da capacidade de oxigenação epitelial de $5,6 \%$, um aumento do polimegatismo em $22 \%$ e uma indução de microcistos epiteliais. A reversão do afinamento estromal foi o único efeito não observado após 33 dias sem o uso de $\mathrm{LC}^{18}$. No trabalho que estudou o uso crônico de LC em olhos míopes com média de uso de 13,45 $\pm 6,42$ meses, observou-se uma redução significativa da espessura corneana de 30 a $50 \mu \mathrm{m}$ e uma maior curvatura na ceratometria quando comparado aos olhos sem uso de $\mathrm{LC}^{(15)}$. Não foi encontrada relação entre a espessura central corneana e o grau da miopia neste estudo ${ }^{15}$. Devido ao caráter retrospectivo do nosso estudo, não foi possível colher dados a respeito do tempo de uso de LC de todos os pacientes e estabelecer uma correlação com os demais parâmetros corneanos.

Alguns trabalhos sugerem uma diferença étnica na espessura central corneana entre asiáticos e caucasianos míopes, sendo menor nos primeiros, semelhante aos relatos em populações normais. Outras variações étnicas na espessura central corneana têm sido relatadas. Americanos de origem africana têm em média córneas mais finas comparadas com caucasianos $^{(19-20)}$. Um estudo mostrou uma espessura central corneana média em caucasianos de $550 \mu \mathrm{m}^{16}$. Outro reportou uma espessura central média de 505,9 $\mu \mathrm{m}$ em uma população normal da zona rural do sul da Índia ${ }^{(21)}$.

Estudos comparando o Pentacam ${ }^{\circledR}$ com outros aparelhos para a medida da espessura corneana mostraram boa correlação entre eles. Um estudo reportando a medida da espessura central corneana com o Pentacam ${ }^{\circledR}$ foi semelhante à obtida com o paquímetro de reflectometria de baixa coerência e com o paquímetro ultra-sônico ${ }^{(4)}$. Outro estudo também achou medidas comparáveis entre o Pentacam ${ }^{\circledR}$, o Orbscan ${ }^{\circledR}$ e o paquímetro ultrasônico ${ }^{(5)}$. Igualmente as medidas de espessura central com os aparelhos Pentacam ${ }^{\circledR}$, Orbscan ${ }^{\circledR}$ e paquimetria por interferometria de coerência parcial também se correlacionam bem ${ }^{(6)}$.

No presente estudo, observou-se, na comparação entre míopes e hipermétropes, uma maior profundidade $(\mathrm{p}<0,001)$ e volume de câmara anterior $(\mathrm{p}<0,001)$ nos míopes. A comparação dos míopes e hipermétropes com ametropias semelhantes mostrou a mesma tendência. A análise da subdivisão dos míopes mostrou que o volume de câmara anterior e a profundidade de câmara anterior foram maiores no grupo com ametropias entre $-6,1$ a -9 D.

Existem poucos dados na literatura a respeito de medidas do segmento anterior. Os valores de câmara anterior são importantes no planejamento cirúrgico para cálculo de lente intra-ocular ${ }^{(22-23)}$ ou para implantação de lentes fácicas ${ }^{(24-25)}$. Num estudo prospectivo, que avaliou medidas do segmento anterior em indivíduos saudáveis com o aparelho Pentacam ${ }^{\circledR}$, o volume de câmara anterior médio foi de $160,4 \pm 36,81 \mathrm{~mm}^{3} \mathrm{e}$ da profundidade de câmara anterior de 2,93 $\pm 0,36 \mathrm{~mm}$. Houve uma excelente correlação entre o volume e a profundidade de câmara anterior $(\mathrm{r}=0,92)^{(26)}$.
A comparação de medidas de câmara anterior realizadas pelo Pentacam ${ }^{\circledR}$, Orbscan ${ }^{\circledR}$ e AC-Master ${ }^{\circledR}$ (interferometria de coerência parcial) em olhos saudáveis (equivalente esférico médio de $-2,15 \mathrm{D})$, mostrou médias de profundidade de $3,15 \pm$ $0,28 \mathrm{~mm} ; 3,12 \pm 0,27 \mathrm{~mm} ; 3,32 \pm 0,24 \mathrm{~mm}$ respectivamente. Houve uma correlação melhor entre as medidas do Pentacam ${ }^{\circledR}$ e do Orbscan ${ }^{\circledR}(\mathrm{r}=0,96)$. Os três métodos se mostraram excelentes métodos de não-contato para medida de profundidade de câmara anteiror ${ }^{(6)}$. A medida da profundidade de câmara anterior foi semelhante ao nosso estudo, onde para um equivalente esférico de 0 a $-3 \mathrm{D}$ a média da profundidade foi de $3,15 \pm$ $0,28 \mathrm{~mm}_{\text {com o Pentacam }}^{\circledR}$.

O sistema Scheimpflug mostrou que a profundidade da câmara anterior diminuiu e a espessura do cristalino aumentou com a idade, estabelecendo uma correlação negativa entre estas variáveis ${ }^{(27)}$. $\mathrm{O}$ aumento progressivo da espessura do cristalino com o envelhecimento resulta em uma diminuição progressiva da profundidade da câmara anterior ${ }^{(27)}$. Neste estudo, a comparação da média de idade entre os míopes e hipermétropes foi significativa (32,61 anos versus 40,30 anos; $\mathrm{p}<0,001)$, mostrando que os míopes eram mais jovens que os hipermétropes. Talvez a mudança do cristalino com a idade possa influenciar o nosso achado de menor medida de câmara anterior nos hipermétropes em relação aos míopes, mas a espessura do cristalino não foi avaliada neste estudo. Não houve diferença estatisticamente significativa quanto à idade entre os subgrupos dos míopes. $\mathrm{O}$ achado de menor profundidade e volume de câmara anterior no grupo 4 em relação aos demais subgrupos provavelmente se deve ao menor número de pessoas neste grupo.

A idade não parece influenciar a espessura corneana. A diferença de idade entre o grupo dos míopes e hipermétropes foi significativa no presente estudo, mas foi observado em um trabalho que a paquimetria não se altera significativamente ao longo dos anos (faixa etária de 5 a 64 anos) ${ }^{(28)}$. Um outro estudo mostrou que a espessura central corneana média foi de $529 \mu \mathrm{m}$ em crianças, de $533 \mu \mathrm{m}$ em adultos e de $527 \mu \mathrm{m}$ em idosos, sem diferença significativa entre os grupos ${ }^{(29)}$.

\section{CONCLUSÃO}

Concluímos que o Pentacam ${ }^{\circledR}$ é um aparelho de não-contato muito útil na avaliação do segmento anterior. Quanto às variáveis analisadas, observou-se um menor volume corneano ao redor do ápice principalmente nos $3 \mathrm{~mm}, 5 \mathrm{~mm}$ e $7 \mathrm{~mm}$ em pessoas míopes. A profundidade e o volume de câmara anterior se mostraram maiores em míopes e a paquimetria menos espessa nesta população.

\section{ABSTRACT}

Purpose: To analyze the anterior segment of refractive surgery candidates and establish the variability pattern in this population regarding corneal volume, anterior chamber volume and 
depth and corneal thickness, using the noncontact three dimensional analyzer Pentacam ${ }^{\circledR}$. Methods: A retrospective study of 297 eyes of 149 patients was conducted using Pentacam ${ }^{\circledR}$. According to the spherical equivalent value two groups were created, myopia $(\mathrm{n}=242$ eyes) and hyperopia $(\mathrm{n}=55$ eyes), to analyze the variables of corneal volume, anterior chamber volume and depth and corneal thickness. Results: The mean values of the myopia group were: corneal total volume $59.37 \pm 3.51 \mathrm{~mm}^{3}$, corneal volume at $3 \mathrm{~mm} 3.87 \pm 0.23 \mathrm{~mm}^{3}$, at $5 \mathrm{~mm} 11.31 \pm 0.67 \mathrm{~mm}^{3}$, and at $7 \mathrm{~mm} 24.30 \pm 1.43 \mathrm{~mm}^{3}$, anterior chamber volume 198.74 $\pm 32.40 \mathrm{~mm}^{3}$, anterior chamber depth $3.19 \pm 0.28 \mathrm{~mm}$ and corneal thickness $533.33 \pm 33.47 \mu \mathrm{m}$. In the hyperopia group, the mean total corneal volume was $60.77 \pm 3.31 \mathrm{~mm}^{3}$, corneal volume at 3 $\mathrm{mm}$ from the apex was $4.01 \pm 0.20 \mathrm{~mm}^{3}$, at $5 \mathrm{~mm}$ was $11.73 \pm$ $0.58 \mathrm{~mm}^{3}$, and at $7 \mathrm{~mm}$ was $25.09 \pm 1.21 \mathrm{~mm}^{3}$, the anterior chamber volume was $146.61 \pm 32.86 \mathrm{~mm}^{3}$, the anterior chamber depth was $2.76 \pm 0.38 \mathrm{~mm}$ and the corneal thickness was $550.52 \pm 29.49 \mu \mathrm{m}$. The difference between the groups was significant for all variables $(\mathrm{p}<0.05)$. Conclusion: We observed in this study that patients with myopia had lesser mean corneal volume and pachymetry, and greater anterior chamber depth and volume compared with hyperopic patients.

Keywords: Corneal topography; Myopia/surgery; Hyperopia/ surgery; Anterior eye segment

\section{REFERÊNCIAS}

1. Nishida T. Cornea. In: Krachmer JH, Mannis MJ, Holland EJ, editors. Cornea and external disease: clinical diagnosis and management. St. Louis: Mosby-Year Book; 1997. p.3-27.

2. Alves MR, Kara-José N. Edema corneano. In: Belfort Jr R, Kara-José N. Córnea: clínica, cirúrgica. São Paulo: Roca; 1997. p.17-25.

3. Ehlers N, Hjortdal J. Corneal thickness: measurement and implications. Exp Eye Res. 2004;78(3):543-8.

4. Barkana Y, Gerber Y, Elbaz U, Schwartz S, Ken-Dror G, Avni I, Zadok D. Central corneal thickness measurement with the Pentacam ${ }^{\circledR}$ Scheimpflug system, optical low-coherence reflectometry pachymeter, and ultrasound pachymetry. J Cataract Refract Surg. 2005;31(9):1729-35.

5. Amano S, Honda N, Amano Y, Yamagami S, Miyai T, Samejima T, et al. Comparison of central corneal thickness measurements by rotating Scheimpflug camera, ultrasonic pachymetry, and scanning-slit corneal topography. Ophthalmology. 2006;113(6):937-41.

6. Buehl W, Stojanac D, Sacu S, Drexler W, Findl O. Comparison of three methods of measuring corneal thickness and anterior chamber depth. Am J Ophthalmol. 2006;141(1):7-12.

7. Pentacam ${ }^{\circledast}$ - Instruction Manual - Measurement and evaluation system for the anterior segment of the eye. Alemanha: Oculus, Wetzlar; s.d.
8. Suzuki H, Takahashi H, Hori J, Hiraoka M, Igarashi T, Shiwa T. Phacoemulsification associated corneal damage evaluated by corneal volume. Am J Ophthalmol. 2006;142(3):525-8.

9. Kirchhoff A, Böhm H, Schäferhoff C, Beck R, Guthoff R. [Measuring corneal volume. A possible quality parameter in cataract surgery?] Ophthalmologe. 2001;98(4):402-5. Ger.

10. Chang SW, Tsai IL, Hu FR, Lin LL, Shih YF. The cornea in young myopic adults. Br J Ophthalmol. 2001;85(8):916-20.

11. Von Bahr G. Corneal thickness: its measurement and changes. Am J Ophthalmol. 1956;42(2):251-66.

12. Tokoro T, Hayashi $\mathrm{K}$, Muto $\mathrm{M}$, et al. Central corneal thickness in high myopia. Folia Ophthalmol Jpn. 1976;27:610.

13. Tanaka HM, Mori ES, Maia NCF, Juliano Y, Campos M. Espessura corneana na alta miopia. Arq Bras Oftalmol. 1996;59(3):295-8.

14. Fam HB, How AC, Baskaran M, Lim KL, Chan YH, Aung T. Central corneal thickness and its relationship with myopia in Chinese adults. Br J Ophthalmol. 2006;90(12):1451-3.

15. Liu Z, Pflugfelder SC. The effects of long-term contact lens wear on corneal thickness, curvature, and surface regularity. Ophthalmology. 2000;107(1):105-11.

16. Price FW, Koller DL, Price MO. Central corneal pachymetry in patients undergoing laser in situ keratomilieusis. Ophthalmology. 1999;106(11):2216-20.

17. Pedersen L, Hjortdal J, Ehlers N. Central corneal thickness in high myopia. Acta Ophthalmol Scand. 2005;83(5):539-42.

18. Holden BA, Sweeney DF, Vannas A, Nilsson KT, Efron N. Effects of longterm extended contact lens wear on the human cornea. Invest Ophthalmol Vis Sci. 1985;26(11):1489-501.

19. Aghaian E, Choe JE, Lin S, Stamper RL. Central corneal thickness of Caucasians, Chinese, Hispanics, Filipinos, African Americans, and Japanese in a glaucoma clinic. Ophthalmology. 2004;111(12):2211-9.

20. Yo C, Ariyasu RG. Racial differences in central corneal thickness and refraction among refractive surgery candidates. J Refract Surg. 2005;21(2):194-7.

21. Vijaya L, George R, Paul PG, Baskaran M, Arvind H, Raju P, et al. Prevalence of open-angle glaucoma in a rural south Indian population. Invest Ophthalmol Vis Sci. 2005;46(12):4461-7.

22. Olsen T, Corydon L, Gimbel H. Intraocular lens power calculation with an improved anterior chamber depth prediction algorithm. J Cataract Refract Surg. 1995;21(3):313-9.

23. Holladay JT, Gills JP, Leidlein J, Cherchio M. Achieving emmetropia in extremely short eyes with two piggyback posterior chamber intraocular lenses. Ophthalmology. 1996;103(7):1118-23.

24. Allemann N, Chamon W, Tanaka HM, Mori ES, Campos M, Schor P, Baïkoff G. Myopic angle-supported intraocular lenses: two-year follow-up. Ophthalmology. 2000;107(8):1549-54.

25. Mimouni F, Colin J, Koffi V, Bonnet P. Damage to the corneal endothelium from anterior chamber intraocular lenses in phakic myopic eyes. Refract Corneal Surg. 1991;7(4):277-81

26. Rabsilber TM, Khoramnia R, Auffarth GU. Anterior chamber measurements using Pentacam ${ }^{\circledR}$ rotating Scheimplug camera. J Cataract Refract Surg. 2006; 32(3):456-9.

27. Grandberg L, Forseto AS, Souza RF, Nosé RM, Nosé W. Avaliação do envelhecimento do cristalino em olhos normais. Arq Bras Oftalmol. 2001;64 (5):443-7.

28. Leoratti MC, Zampar HV, Costa C, Campos M. Study of ocular aberrations and aging. In: Refractive Surgery Subspecialty Day, 2006. Las Vegas, USA: American Academy of Ophthalmology; 2006.

29. Doughty MJ, Laiquzzaman M, Müller A, Oblak E, Button NF. Central corneal thickness in European (white) individuals, especially children and the elderly, and assessment of its possible importance in clinical measures of intra-ocular pressure. Ophthalmic Physiol Opt. 2002;22(6):491-504. 